\title{
DRG-Einführung
}

\section{in Deutschland: Anspruch,}

Wirklichkeit und

Anpassungsbedarf

aus gesundheits-

\section{ökonomischer Sicht}

Andreas Beivers und Annika Emde

$1.1 \quad$ Wie alles begann - 5

1.2 Einführung des DRG-Systems: Ziele und Umsetzung - 6

1.2.1 Das Ziel der wirtschaftlicheren Versorgung der Bevölkerung mit stationären Leistungen - 8

1.2.2 Das Ziel der Transparenz über Leistungen und Kosten der Krankenhäuser - 10

1.2.3 Das Ziel der Stabilisierung der GKV-Ausgaben durch Erschließung von Wirtschaftlichkeitsreserven - 11

1.2.4 Das Ziel der Verkürzung der Verweildauer der Patienten im Krankenhaus - 11

1.2.5 Das Ziel der Förderung des Wettbewerbs der Krankenhäuser untereinander - 13

1.2.6 Das Ziel der Förderung des Strukturwandels - 15

1.3 Herausforderungen für die Zukunft: Ordnungspolitisch sinnvolle Anpassungen des DRG-Systems - 16

1.3.1 Mangelnde Finanzierung der Investitionskosten - 17

1.3.2 Ambulant und stationär - 18 
1.3.3 Vorhaltung - 19

1.3.4 Indikationsqualität vs. Mengensteuerung - 20

1.4 Ausblick - 20

Literatur - 21 


\section{- Zusammenfassung}

17 Jahre nach der formalen DRG-Einführung in Deutschland steht das deutsche Fallpauschalensystem unter gesellschaftlichem wie politischem Druck. Die Akteure beurteilen die Bilanz der DRG-Einführung äußerst unterschiedlich. Dieser Beitrag wird die zu Beginn der DRG-Einführung gesteckten Ziele auf ihre Zielerfüllung hin untersuchen. Dabei werden aktuelle Schieflagen und Fehlanreize beispielsweise im Bereich der Mengensteuerung, der Qualitätsmessung wie auch der intersektoralen Zusammenarbeit sowie des ungeordneten Strukturwandels im Krankenhausmarkt angesprochen und dargestellt. Es lassen sich dabei ordnungspolitisch sinnvolle Anpassungen des DRG-Systems ableiten, wie beispielsweise Ansätze zur Finanzierung der Investitionskosten, neue Möglichkeiten der ambulant-stationären Zusammenarbeit, die Finanzierung von Vorhalteleistungen und eine verstärkte Steuerung der Versorgung durch Indikationsqualität. Capitation-Modelle stellen in diesem Zusammenhang eine zukunftsweisende Vergütungsoption dar, ebenso wie ein Appell für mehr preisliche Steuerung.

17 years after the formal introduction of DRGs in Germany, the German fee-per-case system is under social and political pressure. The stakeholders assess the results of the DRG introduction extremely differently. This paper examines the goals set at the start of the DRG introduction with regard to their fulfilment. The authors address current imbalances and misplaced incentives, for example in the areas of volume control, quality measurement, inter-sectoral cooperation and disordered structural change in the hospital market. Thus, appropriate regulatory adjustments to the DRG system can be derived, such as approaches to the financing of investment costs, new possibilities for a cooperation of outpatient and inpatient sector, the funding of reserve capacities and increased control of care through indication quality. In this context, capitation models represent a forward-looking payment option as well as an appeal for better price control.

\subsection{Wie alles begann}

Heute, d. h. im Jahr 2020 - und damit 17 Jahre nach der formalen DRG-Einführung in Deutschland - steht das deutsche Fallpauschalensystem unter gesellschaftlichem wie politischem Druck. Die Herausrechnung der Pflegekosten ist nicht das erste, aber ein sehr deutliches Zeichen einer gesundheitspolitischen Willensbekundung, das DRG-System neu zu justieren - oder gegebenenfalls abzuschaffen.

Aber zunächst ein Blick zurück: Ab 1967 begann die durch Robert Barclay Fetter und John Devereaux Thompson eingeleitete Entwicklung der Diagnosis Related Groups (DRGs) an der Yale-Universität. Bis zur verpflichtenden Einführung der DRGs durch das Fallpauschalengesetz und die Fallpauschalenverordnung regelte die Bundespflegesatzverordnung (BPflV) die Details der pflegesatzfähigen Kosten der Krankenhausvergütung ${ }^{1}$. Mit der BPflV 1995 wurde der zuvor angewandte Pflegetag als alleinige Abrechnungseinheit aufgegeben und durch Abteilungspflegesätze sowie Fallpauschalen und Sonderentgelte in Verbindung mit Basispflegesätzen abgelöst (Neubauer 1993, 2002a). Bereits im Herbst 1986 legte das damalige Bundesministerium für Arbeit und Soziales die Ergebnisse des von der Beratungsfirma Ernst \& Whinney durchgeführten Gutachtens zur Einführung von DRGs in Deutschland vor (Ernst \& Whinney 1986; Neubauer und Unterhuber 1987). Zur Anwendung eines DRG-Systems fehlte es jedoch damals an einer einheitlichen Codierung. So wurde ein fünfstelliger Diagnoseschlüssel und ein Prozedurencode für die DRG-Gruppierung vorausgesetzt (Neubauer 1993; Neubauer und Unterhuber 1987). Die

\footnotetext{
Voll- und teilstationäre Krankenhausleistungen, insbesondere ärztliche Behandlung, Krankenpflege, Versorgung mit Arznei-, Heil- und Hilfsmitteln, die für die Versorgung im Krankenhaus notwendig sind, sowie Unterkunft und Verpflegung (Neubauer 1993; Neubauer 2002b).
} 
Schätzung der Beraterfirma ging von einem Zeitbedarf von zehn Jahren aus, bis deutsche Krankenhäuser diese Voraussetzungen erfüllen könnten.

Neben den DRGs wurde 1988/89 zusätzlich das mit den DRGs konkurrierende System der Patient Management Categories (PMCs) auf seine Übertragbarkeit auf Deutschland untersucht. Das Ergebnis dieser Analyse führte zu der Aussage, dass die vorliegenden PMCs auf deutsche Krankenhäuser übertragbar, jedoch in der Breite nur nach Entwicklung bzw. Übernahme eines entsprechenden Prozedurencodes möglich sind (BMG 1990; Neubauer et al. 1992). Schließlich thematisierte der Sachverständigenrat für die Konzertierte Aktion im Gesundheitswesen 1992 in seinem Gutachten den Bedarf für ein leistungsorientiertes Krankenhausvergütungssystem (SVR 1992). Mit der Bundespflegesatzverordnung von 1995 wurden dann vornehmlich für den Bereich der Chirurgie rund 80 Fallpauschalen und etwa 160 Sonderentgelte eingeführt. Damit war zwar ein Paradigmenwechsel in der Krankenhausvergütung eingeleitet, aber noch lange nicht auf breiter Ebene verankert (Neubauer 2003b; Roeder et al. 2007).

Erst mit dem GKV-Gesundheitsreformgesetz im Jahr 2000 wurde die Einführung eines flächendeckenden Fallpauschalensystems in Deutschland, kurz G-DRGs, beschlossen. GDRG (German DRG) ist dabei die Bezeichnung für die deutsche Adaptation des australischen DRG-Systems (AR-DRG). Dass ein DRG-System ins Auge gefasst wurde, war nur folgerichtig, da die DRG-Diskussion in fast allen westeuropäischen Ländern bereits zehn Jahre zuvor geführt worden war (Kimberly und de Pouvourville 1993; Neubauer 1993, 2003b).

So sollten die G-DRGs - auch ordnungspolitisch betrachtet - eine grundlegende Neuausrichtung der Krankenhausvergütung darstellen und das ehemals vorherrschende Selbstkostendeckungsprinzip, in dem Krankenhäuser individuelle Gründe für bestimmte Kostenstrukturen in die Budgetvereinbarungen mit einbringen konnten, endgültig ablösen (Neubauer 2003b; Beivers 2010). Schon damals - ei- nige Jahre vor der offiziellen G-DRG-Einführung im Jahr 2003 - war bekannt, dass der deutsche Krankenhausmarkt vor einer nötigen Strukturanpassung stand (Neubauer 2003a; Beivers und Waehlert 2018). Die Einführung eines neuen Abrechnungssystems auf Bundesebene sollte diese in den Bundesländern vor Ort - u. a. durch die Entstehung von Konvergenzgewinnern und -verlierern - vorantreiben, wohlwissentlich, wie schwierig Strukturanpassungen der regionalen Politik vor Ort umzusetzen sind. Nicht zuletzt bedingt durch diese Entwicklungen befindet sich der deutsche Krankenhausmarkt seit einigen Jahren in einem Wandel (Beivers und Waehlert 2018).

\subsection{Einführung des DRG-Systems: Ziele und Umsetzung}

Offizielles Hauptziel der Einführung des GDRG-Systems war es, durch die leistungsgerechte und transparente Vergütung eine (i) wirtschaftlichere Versorgung der Bevölkerung mit stationären Leistungen zu ermöglichen, (ii) mehr Transparenz über Leistungen und Kosten der Krankenhäuser zu erhalten sowie (iii) die Ausgaben der GKV durch Erschließung von Wirtschaftlichkeitsreserven zu stabilisieren. Daraus abgeleitet erwartete man sich (iv) eine Verkürzung der Verweildauer der Patienten im Krankenhaus, (v) mehr Wettbewerb der Krankenhäuser untereinander sowie (vi) eine Förderung des Strukturwandels. Über allem aber stand das Prinzip: "Geld folgt Leistung“ (Friedrich et al. 2010; Tuschen 2007).

Wie die diagnoseorientierten Fallpauschalen das deutsche Gesundheitssystem verändert haben und ob es zu den gewünschten Effekten kam, ist Gegenstand einer breiten sowohl gesellschaftlichen als auch wissenschaftlichen Debatte. Die Bilanz fällt dabei - je nach Standpunkt - sehr unterschiedlich aus. Einzelne Akteure warnten schon bei Einführung des Systems vor dramatischen Fehlentwicklungen, hinsichtlich derer sich viele heute auch bestätigt sehen: So herrscht nach Aus- 


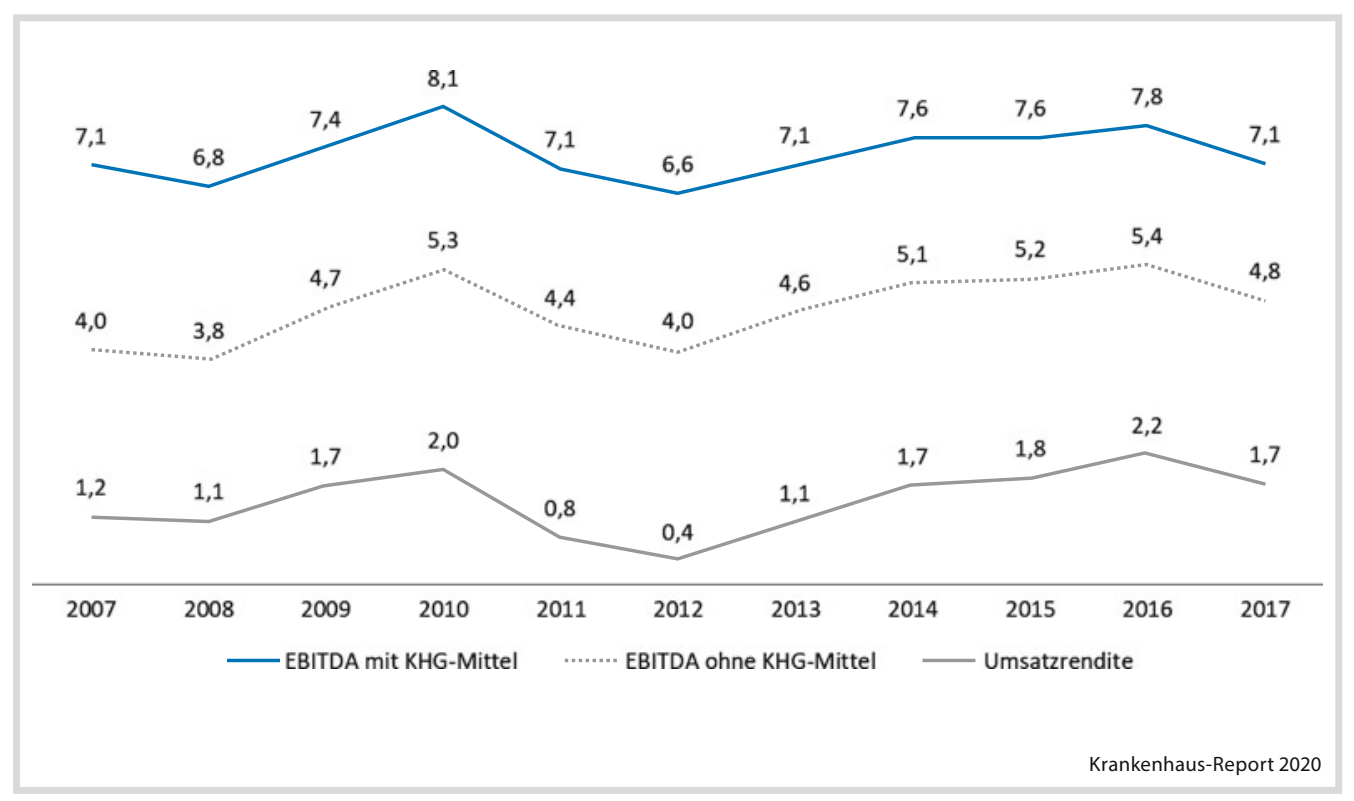

- Abb. 1.1 EBITDA-Margen im Zeitverlauf 2007 bis 2017 in \% (Quelle: Krankenhaus Rating Report 2019; Augurzky et al. 2019) (Anmerkung: Berechnung über das fusionierte Krankenhaus)

sagen des Bündnisses „Krankenhaus statt Fabrik $^{\text {2 }}$ in den Krankenhäusern Personalnot sowie Über-, Unter- und Fehlversorgung. Bei Entscheidungen über Behandlungen und die Dauer des Krankenhausaufenthaltes sind demnach nicht allein medizinische Kriterien entscheidend, sondern auch, was sich gewinnbringend abrechnen lässt. Immer mehr Krankenhäuser werden deswegen privatisiert, so das Bündnis Krankenhaus statt Fabrik (2018). Auch der deutsche Pflegerat hat u. a. 2009 in einer Art Zwischenbilanz eine dringliche Neujustierung erkannt, v. a. in puncto der Abbildung der Pflegelast und der Pflegequalität im Fallpauschalensystem (Müller 2009). Von Seiten der Ärzteschaft sowie des Deutschen Ethikrats gab und gibt es zum Teil heftige Widerstände gegen das Fallpauschalensystem (siehe

2 Das Bündnis "Krankenhaus statt Fabrik" ist ein Zusammenschluss von ver.di-Landesfachbereichen, dem Verein demokratischer Ärztinnen und Ärzte, attac, der Soltauer Initiative, der Gesellschaft für Psychotraumatologie, Traumatherapie und Gewaltforschung (GPTG) sowie Persönlichkeiten aus der Politik. u. a. Roeder et al. 2009; Kapitel III „Medizin“; Deutscher Ethikrat 2016 oder Dieterich et al. 2019).

Aus gesundheitsökonomischer Sicht gibt es aber auch andere Stimmen. So verdient demnach die DRG-Einführung Lob und Anerkennung und ist im Kern eine Erfolgsgeschichte (v. Stackelberg 2009; Baum 2009; Leber und Scheller-Kreinsen 2014; Beivers 2018, 2019b). Wesentliche Ziele der DRG-Einführung sind erreicht worden. Dies ist zum großen Teil der Arbeit des InEK zu verdanken, welches national wie auch international hohes Ansehen genießt. Es gibt wohl kein anderes Fallpauschalensystem, das in diesem Detaillierungsgrad und mit dieser Gründlichkeit kalkuliert ist (Augurzky et al. 2018a). In Hinblick auf Transparenz- und Wirtschaftlichkeitsziele hat das G-DRG-System daher eine weitgehend positive Bilanz aufzuweisen. So hat der Wettbewerb zwischen den Kliniken zugenommen (Waehlert et al. 2015; Beivers und Waehlert 2018), unter Beibehaltung einer respektablen Wirtschaftlichkeit (Leber und Scheller-Kreinsen 2014), wie u. a. die Darstellung der EBIT- 
DA-Margen ${ }^{3}$ deutscher Kliniken im Zeitverlauf ab dem Jahr 2007 zeigt (• Abb. 1.1).

Die unterschiedlichen Wahrnehmungen geben einen ersten Eindruck der kontroversen und nicht immer sachlich geprägten Diskussion. Neben den unterschiedlichen, zum Teil auch politischen und interessengeleiteten heterogenen Ansichten und Einstellungen trägt sicherlich auch die zunehmende Komplexität des DRG-Systems - bedingt u. a. durch eine Vielzahl von Reformen - dazu bei, dass das System für Nicht-Experten immer schwieriger zu durchschauen ist (Dieterich et al. 2019). Diese wahrgenommene "Intransparenz" des DRG-Systems - als eine Art Nebenwirkung des deutschen „100-Prozent-Ansatzes“ - erschwert eine sachliche Auseinandersetzung mit der Thematik. Aus diesem Grund untersucht dieser Beitrag die einzelnen, zu Beginn gesetzten Ziele analytisch und empirisch, um eine Bilanz zu ziehen. Daraus können dann bei fehlender Zielerreichung oder Fehlentwicklungen fundierte Verbesserungsvorschläge abgeleitet werden.

\subsubsection{Das Ziel der wirtschaftlicheren Versorgung der Bevölkerung mit stationären Leistungen}

In diesem Kontext gilt es zunächst zu klären, was unter einer „wirtschaftlichen Versorgung“ zu verstehen ist. Man kann hier die Mikro- und die Makroebene der Betrachtung unterscheiden. Sicherlich ist es auf Mikroebene - sprich auf der Ebene des einzelnen Krankenhauses durch die Fallpauschalen zu einem deutlichen

\footnotetext{
Beim EBITDA handelt es sich um den Ertrag aus dem operativen Geschäft, d.h. die Erlöse nach Abzug von Personal- und Sachkosten. Die Abkürzung steht für „Earnings before interest, taxes, depreciation, and amortization". Es ist eine betriebswirtschaftliche Kennzahl, die relativ gut den Cash Flow eines Unternehmens approximiert. Die EBITDA-Marge ist das Verhältnis von EBITDA zu Gesamterlösen (Augurzky et al. 2018a).
}

Anreiz gekommen, Patienten schneller (d.h. mit einer geringeren Verweildauer) und kostensparender zu behandeln (Geissler et al. 2010; Neubauer 2003a), da die Vergütung nicht mehr pro Tag, sondern pro Fall erfolgt. Demzufolge werden medizinische und pflegerische Entscheidungen auch in einem betriebswirtschaftlichen Kontext getroffen (Dieterich et al. 2019). Da im gegenwärtigen DRG-System die Erlöse vor allem über ärztliche Leistungen erzielt werden, wurden über die Jahre hinweg viele Ärzte eingestellt. Andere Personalgruppen sind bzw. waren weniger erlösrelevant und wurden $\mathrm{zu}$ mindest in den ersten Jahren nach der DRGEinführung abgebaut (Augurzky et al. 2019). Zusammen mit notwendigen Prozessoptimierungen zur Verweildauerreduktion und Outsourcing krankenhausferner Dienste agierten viele Kliniken damit insgesamt wirtschaftlicher als zu Zeiten der Selbstkostendeckung.

Auf der Makroebene stellt sich hingegen die Frage, ob zum einen die Ausgaben für die Behandlung der stationären Patienten gesunken sind (was im Folgenden noch genauer dargestellt wird) und ob es zum anderen $\mathrm{zu}$ einer Steigerung der Produktivität der Krankenhäuser gekommen ist. Ein erster Blick auf die Makroebene zeigt, dass das Marktvolumen der Krankenhäuser insgesamt kontinuierlich angestiegen ist, und zwar seit der Finanzkrise 2009 stärker als das Bruttoinlandsprodukt (• Abb. 1.2).

Auch in Bezug auf die stationären Fallzahlen ist seit 2002 eine jährliche Steigerung der Krankenhauskosten je Fall von 3,2\% bei den Personalkosten und 2,9\% bei den Sachkosten $\mathrm{zu}$ beobachten, wie die Auswertungen des Krankenhaus Rating Reports 2019 zeigen. Das lässt nicht unbedingt mehr Wirtschaftlichkeit vermuten. Bei dem Versuch, die Entwicklung der Produktivität darzustellen, kann ein Vergleich der erlösrelevanten Vergütungspunkte (Casemix) mit den ärztlichen Vollkräften genutzt werden. Entgegen der Annahme einer Produktivitätssteigerung sank der Casemix je ärztliche Vollkraft im Zeitraum 2007 bis 2017 über alle Krankenhäuser um zwei Punkte auf 140, anstatt zu steigen. Lag der Wert im Jahr 


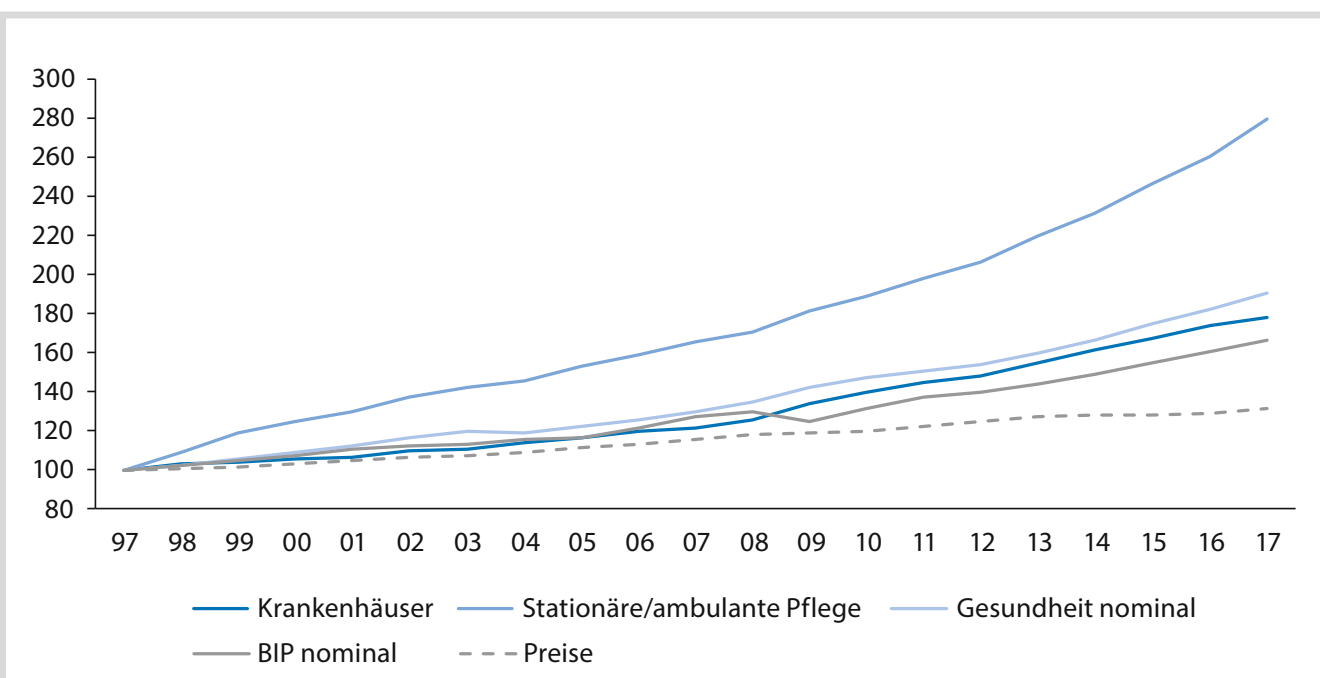

Krankenhaus-Report 2020

- Abb. 1.2 Marktvolumen Änderung 1997-2017 (1997= 100) (Quelle: Krankenhaus Rating Report 2019; Augurzky et al. 2019) (Anmerkung: Gesamtkosten ohne Kosten für das Ausland. Ab 2014 werden Leistungen für Dialysezentren den Arztpraxen zugerechnet)

2009 noch bei 150 Casemixpunkten je ärztliche Vollkraft, ging er im Jahr 2013 auf unter 146 zurück. Nach einem geringfügigen Anstieg im Jahr 2014 sank er 2017 weiter auf 140 Casemixpunkte je ärztlich Vollkraft (• Abb. 1.3) ${ }^{4}$. Unterschieden nach Trägerschaft konnten nur bei privaten Kliniken und Universitätskliniken die Casemixpunkte je ärztliche Vollkraft gesteigert werden. Universitätskliniken liegen aufgrund ihrer Besonderheit bei Forschung und Lehre im Schnitt unter 100 Casemixpunkten je ärztliche Vollkraft (Augurzky et al. 2019).

Betrachtet man hingegen die Pflege im Krankenhaus, ergibt sich ein etwas anderes Bild: Hier scheint es zu einer Produktivitätszunahme gekommen zu sein. So ist nach Auswertungen von Augurzky et al. (2016) im Zeitraum von 2006 bis 2014 die Anzahl der Casemixpunkte je Vollkräfte im Pflegedienst um

4 Für 2017 1.171 Krankenhäuser, davon 26 Unikliniken. Es werden hier nur die Vollkräfte aus allgemeinen Krankenhäusern betrachtet. Die Werte für die Träger "Öffentlich-rechtlich" und „Privat" sind um die Fallzahlen aus den Unikliniken bereinigt worden.
10 bis $20 \%$ gestiegen. Auch die Analysen der Bertelsmann Stiftung weisen in eine ähnliche Richtung: Während eine Pflegevollkraft im Jahr 2003 in Allgemeinkrankenhäusern statistisch noch 57,3 Behandlungsfälle zu betreuen hatte, waren es 2015 schon 64, was 11,6\% mehr Patienten pro Pflegekraft bedeutet (Bertelsmann Stiftung 2017). Aktuelle Auswertungen weisen dabei auch auf trägerspezifische Unterschiede hin (Augurzky et al. 2018a).

Basierend auf diesen Daten kann folglich nicht pauschal davon ausgegangen werden, dass es zu einer wirtschaftlicheren Versorgung der Bevölkerung mit stationären Leistungen in toto gekommen ist. Vielmehr ist auf Mikroebene ein heterogenes Abschneiden im Bereich der Produktivität zwischen den einzelnen Berufsgruppen wie auch den Krankenhäusern zu vermuten. Darauf weisen auch die Ergebnisse des Krankenhaus Rating Reports 2019 hin, wonach beispielsweise große Krankenhäuser typischerweise ein besseres Rating als kleine aufweisen. Aber auch ein hoher Grad an Spezialisierung beeinflusst das Rating positiv. Kliniken in freigemeinnütziger und privater Träger- 

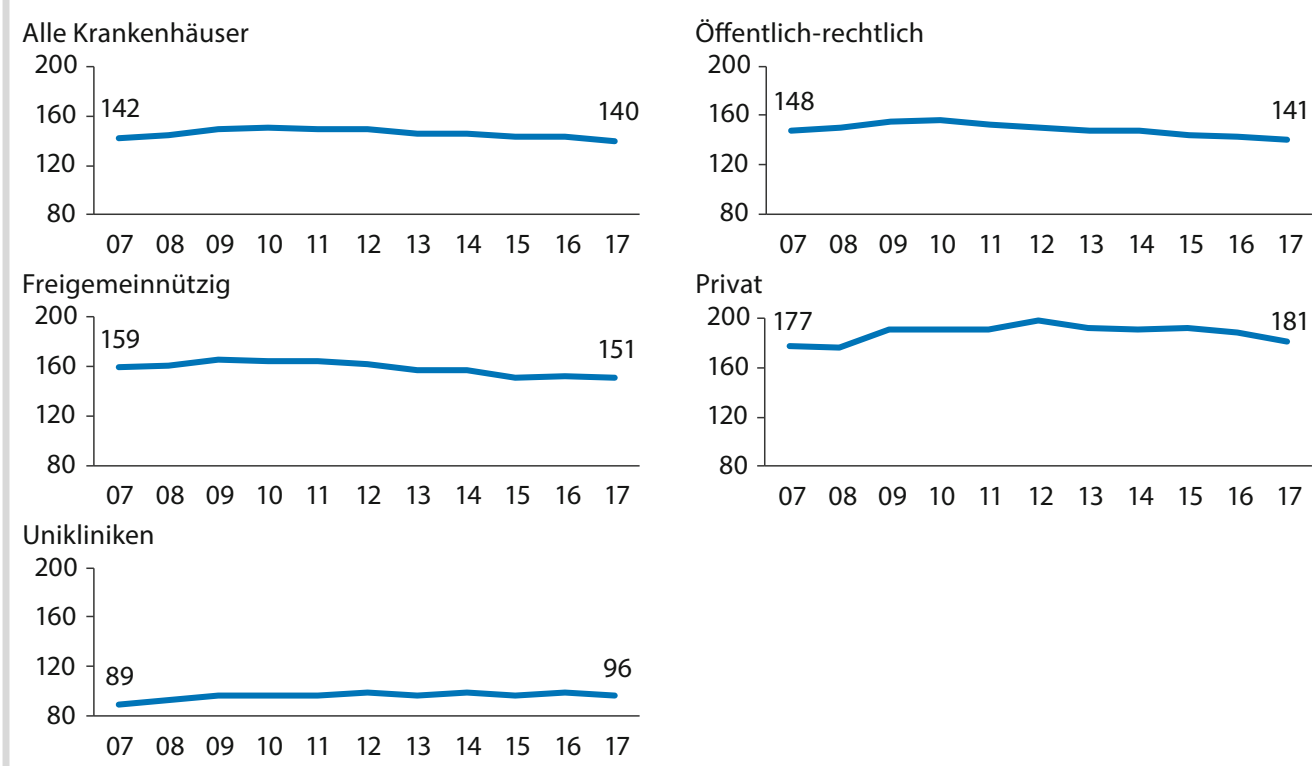

Krankenhaus-Report 2020

- Abb. 1.3 Casemixpunkte je ärztliche Vollkraft nach Trägerschaft (2007 bis 2017) (Quelle: Krankenhaus Rating Report 2019; Augurzky et al. 2019)

schaft schneiden demnach besser ab als öffentlich-rechtliche Kliniken (Augurzky et al. 2019). Grund dafür dürften auch Unterschiede in der Produktivität der Patientenbehandlung sein.

Ein weiterer Aspekt bzgl. der Wirtschaftlichkeit der Versorgung auf der Makroebene ist die Frage, ob Krankenhäuser - bedingt durch das DRG-System - einen Anreiz haben, Patienten auch stationsersetzend (d.h. ambulant oder teilstationär) $\mathrm{zu}$ behandeln. Betrachtet man das wachsende stationäre Behandlungsvolumen, scheint dieses Ziel kaum erreicht worden zu sein. Deswegen ist die ambulant-stationäre Schnittstelle auch oftmals Gegenstand von Prüfungen des Medizinischen Dienstes der Krankenkassen. Der $>$ Abschn. 1.3.2 weiter unten nimmt sich dieser Problematik detaillierter an und zeigt dabei auch Lösungsoptionen auf.

\subsubsection{Das Ziel der Transparenz über Leistungen und Kosten der Krankenhäuser}

Sicherlich hat das DRG-System zu einer Steigerung der Transparenz über die erbrachten Leistungen und die daraus resultierenden Kosten geführt. Standen zur DRG-Einführung knapp 660 DRGs zur Verfügung, sind es 2019 mit rund 1.320 doppelt so viele. Mit über 1.500 Diagnosecodes (Dreistellige ICD-Codes) und knapp 26.000 Prozedurencodes kann jeder stationäre Krankenhausfall beschrieben werden. Die regelmäßigen Auswertungen und bundesweiten Aufstellungen - z. B. im Rahmen der Begleitforschung durch das InEK oder durch das Statistische Bundesamt - schaffen Transparenz über die Leistungen der Krankenhäuser über die reinen Fallzahlen hinaus. Mit Einführung der Verpflichtung zur Veröffentlichung der strukturierten Qualitätsberichte wurde eine zusätzliche Quelle zur Transparenz über Fall- 
und Eingriffszahlen und Qualitätsindikatoren geschaffen, die auch für Patienten über diverse Internet-Plattformen zugänglich ist und verständlich aufbereitet wird.

Fraglich ist hingegen, ob die Transparenz über die Qualität der Leistungserbringung wesentlich gestiegen ist. So handelt es sich - ökonomisch betrachtet - bei den derzeitigen DRGEntgelten um staatlich regulierte Einheitspreise, bei der viele Leistungserbringer kaum einen direkten Anreiz haben, die bestmögliche Qualität sicherzustellen, da sich die Ergebnisqualität der erbrachten Leistung nicht auf ihre Vergütung auswirkt. So haben die DRGs den Nachteil, dass für die Krankenhäuser lediglich die Prozessqualität im Mittelpunkt steht, um mit den kalkulierten Fallpauschalen den Behandlungsprozess finanzieren zu können (vgl. Braun et al. 2008; Geissler et al. 2012; Neubauer und Beivers 2010).

Deshalb ist es angebracht, die Herstellung von Qualität auch über Vergütungsanreize zu steuern, um für die Krankenhäuser einen direkten, monetären Anreiz zu schaffen. Eine direkte Steuerung der Qualität über Vergütungsanreize impliziert jedoch, dass sich die Ergebnisqualität erfassen, messen und bewerten lässt, um dann von den Kostenträgern über entsprechende Entgelte entweder belohnt oder sanktioniert zu werden. Betriebswirtschaftlich betrachtet ist es hingegen die ureigene Aufgabe eines Unternehmens, eine unter Qualitätsaspekten optimale Ressourcenallokation unter der Restriktion beschränkter Finanzmittel $-\mathrm{zu}$ erreichen. In einem aufgrund mangelnder Marktfunktionalität notwendigerweise regulierten und dazu äußerst komplexen Vergütungssystem wie dem DRG-System kann es jedoch passieren, dass die vorherrschenden Preissignale unter dem Aspekt der Versorgungsqualität nicht immer die richtigen Anreize für Krankenhäuser setzen. Das im Status quo regulierte Vergütungs- bzw. Preissystem sollte daher sinnvoll ausgebaut werden und nach Augurzky et al. (2018a) auch um weitere Qualitätsaspekte ergänzt werden. Dabei sollte neben der medizinischen Ergebnisqualität beispielsweise auch die Pflegequalität als weitere Ziel- größe in Betracht gezogen werden (Augurzky et al. 2018a).

\subsubsection{Das Ziel der Stabilisierung der GKV-Ausgaben durch Erschließung von Wirtschaftlichkeitsreserven}

Dieser Punkt kann relativ kurz und deutlich erörtert werden: Betrachtet man die Ausgabendynamik der gesetzlichen Krankenversicherung (GKV) im stationären Bereich pro Versicherten, so ist eindeutig festzustellen, dass das damalige Ziel der Ausgabenreduktion deutlich verfehlt wurde (• Abb. 1.4).

Zwar ist es retrospektiv schwierig abzuschätzen, wie sich die Ausgaben ohne die DRGs entwickelt hätten. Doch kann dieser Befund für sich genommen nicht befriedigen. Aus gesundheitsökonomischer Sicht sollte ein wichtiges Ziel sein, diese Ausgabenentwicklung zu dämpfen. Denn die geburtenstarken Jahrgänge werden schon bald aus dem Erwerbsleben ausscheiden. Der große „Rentenansturm“ ist im Laufe der 2020er-Jahre zu erwarten und mithin auch eine wachsende Nachfrage nach Gesundheitsleistungen, bei einer gleichzeitig relativ sinkenden Grundlohnsumme - und somit relativ schrumpfenden Finanzierungsbasis der gesetzlichen Krankenversicherung (Augurzky und Beivers 2019). Umso wichtiger ist es, die Ausgabenentwicklung bereits heute durch das Vergütungssystem effektiv zu lenken.

\subsubsection{Das Ziel der Verkürzung der Verweildauer der Patienten im Krankenhaus}

Seit 1999 hat sich die durchschnittliche Verweildauer um 26,5\% reduziert (• Abb. 1.5). Dieser Rückgang begann schon vor Einführung des DRG-Systems mit der Einführung der ersten Fallpauschalen. Seit 2004 lässt sich entgegen den Erwartungen sogar eine leichte Verlangsa- 


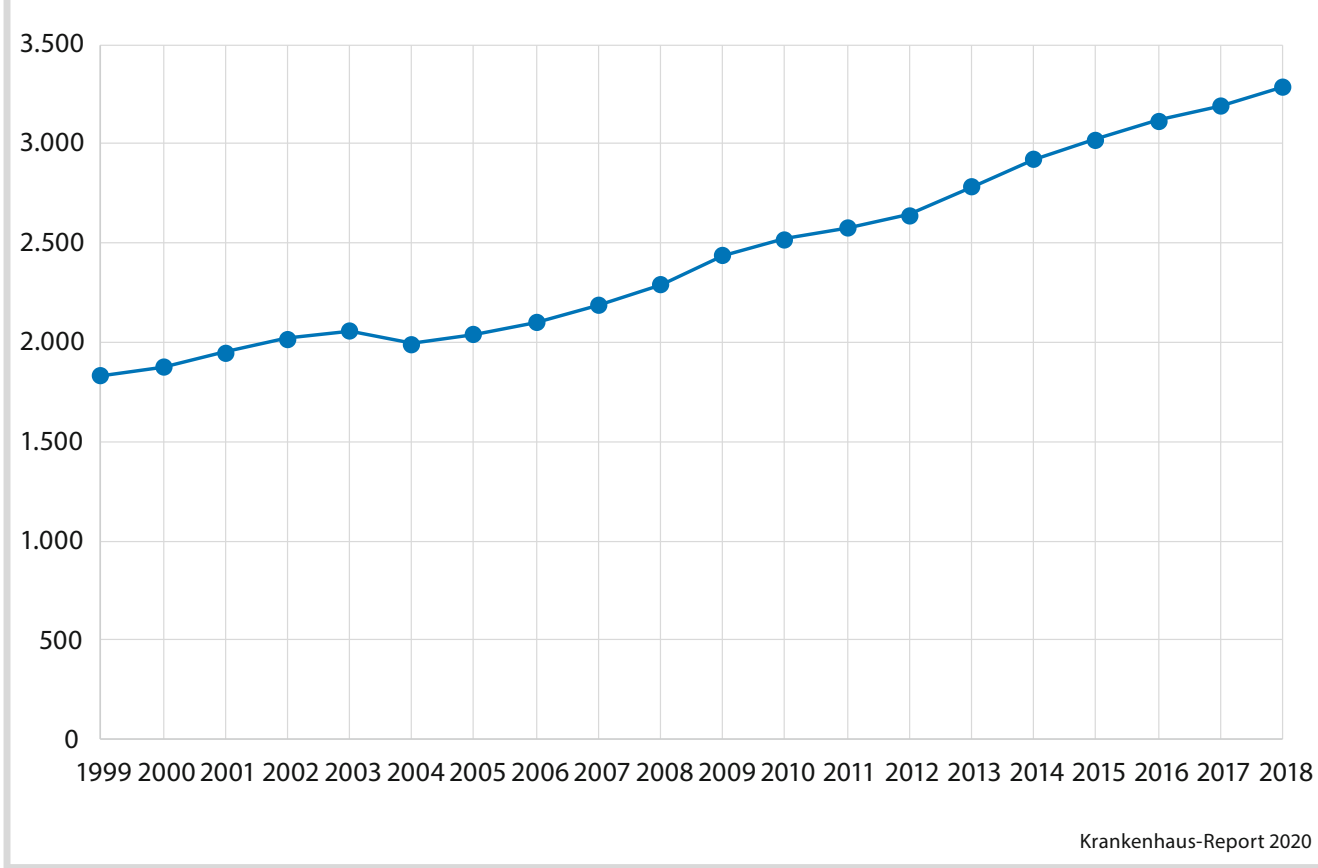

- Abb. 1.4 GKV-Ausgaben Krankenhausbehandlung je Versicherten in Euro (Quelle: BMG 2019, KJ 1-Statistik)

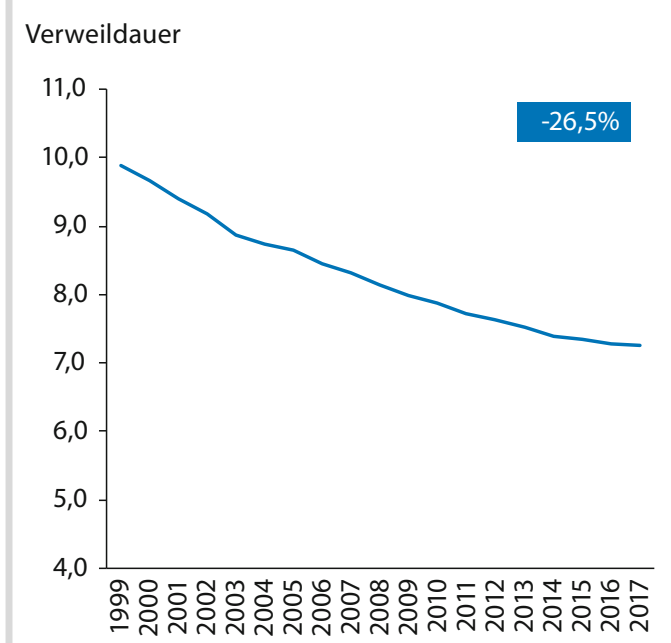

Belegungstage in Mio.

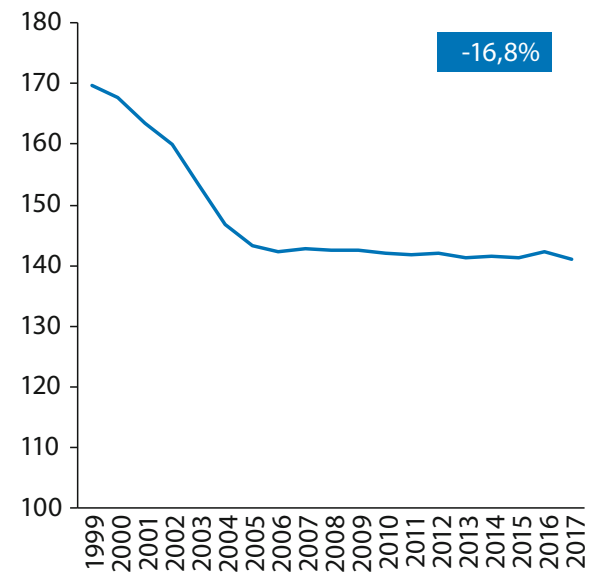

Krankenhaus-Report 2020 Statistisches Bundesamt 2018) 
mung des Rückgangs erkennen. Gründe hierfür könnten sein, dass erstens stationäre Fälle mit kurzer Verweildauer verstärkt ambulant erbracht werden und zweitens der Anteil älterer Patienten mit durchschnittlich längerer Verweildauer zunimmt. Der starke Rückgang der Verweildauer führte zwischen 1999 und 2006 trotz steigender Zahl der Fälle zu einem beachtlichen Rückgang der Belegungstage. Seit 2007 bewegt sich die Zahl der Belegungstage jedoch relativ unverändert bei 142 Mio. Offenbar wurde die rückläufige Verweildauer exakt durch eine steigende Zahl an Fällen kompensiert (Augurzky et al. 2019).

Die gewünschte Verweildauerreduktion ist also zumindest teilweise eingetreten; es ist jedoch - gerade hinsichtlich der Fallzahlsteigerung (zumindest vor dem Krankenhausstrukturgesetz (KHSG)) - zu neuen Anreizen gekommen. Den Anreiz zur Mengenausweitung haben die verschiedensten wissenschaftlichen Studien untersucht (u. a. RWI 2012; Schreyögg 2014; Reifferscheid et al. 2012; Mostert et al. 2012; Lüngen und Büscher 2012). Es ist daher nach Reifferscheid et al. (2012) anzunehmen, dass sich die monetären Anreize der DRG-Vergütungssystematik maßgeblich auf die Entscheidungen des Krankenhausmanagements auswirken. Hinzu kommt, dass es im DRG-System für ein Krankenhaus den Anreiz gibt, mehrere erforderliche Eingriffe nicht im Zuge nur eines Krankenhausaufenthalts, sondern nach medizinischer Möglichkeit auf verschiedene Aufenthalte bzw. Krankenhausfälle zu verteilen.

$\mathrm{Zu}$ einem ganz ähnlichen Ergebnis kommt auch die Studie "Mengenentwicklung und Mengensteuerung stationärer Leistungen" des RWI - Leibniz-Institut für Wirtschaftsforschung, die auf Grundlage der Daten nach $\$ 21$ KHEntgG den Anstieg des Casemix im Zeitraum von 2006 bis 2010 detailliert beleuchtet (RWI 2012). Daher hat sich nicht zuletzt die Gesundheitspolitik via KHSG dieses Themas angenommen und u. a. durch eine gezielte Absenkung von Bewertungsrelationen in einzelnen Indikationsgebieten wie v.a. auch durch den sogenannten Fixkostendegressions- abschlag (FDA) versucht, die Leistungsentwicklung zu steuern. Dies scheint Steuerungseffekte zu zeigen: So ist nun erstmals im Jahr 2018 ein Rückgang des Casemix-Volumens im deutschen Krankenhausmarkt zu verzeichnen.

\subsubsection{Das Ziel der Förderung des Wettbewerbs der Krankenhäuser untereinander}

Der v. a. bis zum Jahr $2009 \mathrm{zu}$ beobachtende Abbau von Krankenhausbetten kann als Folge einer gestiegenen Wettbewerbsintensität interpretiert werden. Auch die Zahl der Krankenhäuser (Institutionskennziffern) verringerte sich seit 1999 um $13,8 \%$ und seit 2003 um $11,6 \%$ (• Abb. 1.6). Parallel zu dieser Entwicklung war gerade zu Beginn der Fallpauschalen-Einführung ein vermehrter Trägerwechsel von Krankenhäusern hin zu privaten, gewinnorientierten Unternehmen zu beobachten (- Abb. 1.7).

Die Privatisierung von Krankenhäusern wird seit Anfang der 1990iger Jahre kontrovers diskutiert. Zur Versachlichung der Debatte wurden in den Jahren 2009, 2012 und 2015 Faktenbücher zur Bedeutung der Krankenhäuser in privater Trägerschaft erstellt (Augurzky et al. 2018a). Sie zeigen u. a. den gestiegenen Wettbewerb der Krankenhausträger auf. Dies dürfte ein Effekt der Fallpauschalen-Einführung sein. Die Analysen verdeutlichen aber auch, dass bei gleichem Ressourceneinsatz private Träger - gemessen in Casemixpunkten - eine höhere Leistungsmenge als andere Träger erreichen. Sicherlich lassen sich daraus keine pauschalen Rückschüsse auf die Versorgungsqualität ableiten. Es zeigt sich darüber hinaus, dass private Krankenhäuser die geringsten Kosten und höchsten Investitionen je Casemixpunkt aufweisen. Seit 2012 stagnieren jedoch die Marktanteile der verschiedenen Trägerschaften weitgehend auf ihrem Niveau von 2011.

Parallel zur Kapazitätsreduktion und zu den Trägerwechseln ging die Bettenauslastung von 
Zahl der Krankenhäuser

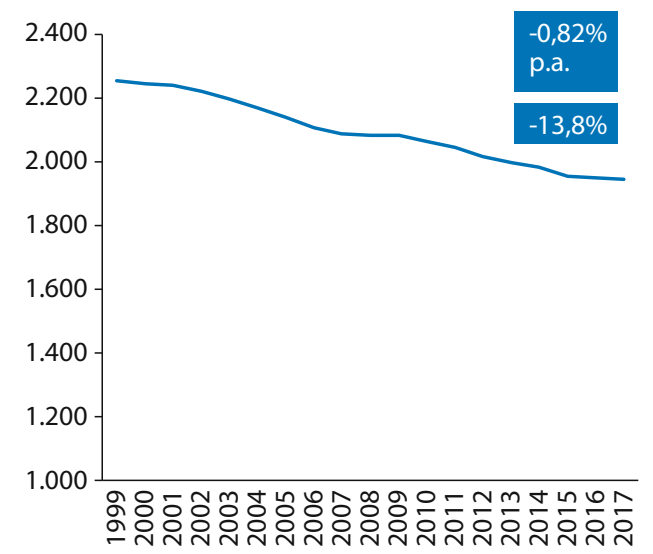

Zahl der Krankenhausbetten, in 1.000

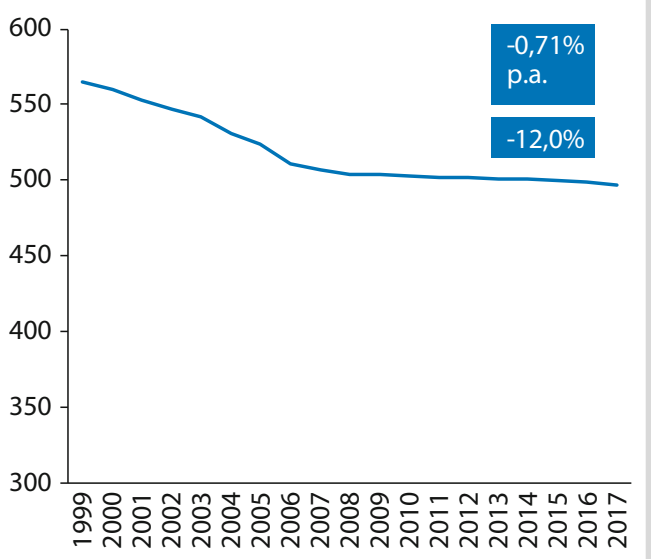

Krankenhaus-Report 2020

- Abb. 1.6 Zahl der Krankenhäuser (nach Institutionskennzeichen) und Krankenhausbetten (1999 bis 2017) (Quelle: Augurzky et al. 2019; Statistisches Bundesamt 2018)

1999 bis 2005 von 81,1 auf 74,9\% zurück. Im Jahr 2017 lag sie bei 77,8\%. Bezogen auf eine maximale Auslastung von $85 \%$ schätzt das RWI daher die Bettenüberkapazität für 2017 auf 8,5 \% (Augurzky et al. 2019). Gemäß dieser Annahme könnten in Bezug auf Krankenhausstandorte rund $16 \%$ geschlossen werden, wenn man die Krankenhausdichte Sachsens zugrunde legte. Das Bundesland Sachsen eignet sich in diesem Zusammenhang deswegen so gut als Benchmark, weil nicht nur die wirtschaftliche Lage der sächsischen Krankenhäuser bundesweit die Beste ist, sondern auch, weil die Kapitalausstattung sowie die Krankenhausstrukturen im innerdeutschen Vergleich als gut zu bezeichnen sind, wenngleich es im internationalen Vergleich trotzdem Verbesserungspotenziale gäbe (Augurzky et al. 2017). Andere Studien verweisen hier $u$. a. auch auf die Niederlande, die hinsichtlich der Bevölkerungszahl und Fläche mit Nordrhein-Westfalen vergleichbar sind, aber rund zwei Drittel weniger Krankenhäuser vorhalten, sowie auf Dänemark, das derzeit eine umfangreiche Zentralisierung seiner Krankenhausversorgung umsetzt (Hacker 2016; Geissler et al. 2010; Bertelsmann Stiftung
2019). Würde eines dieser Länder als Benchmark gewählt, könnte die Krankenhausdichte in Deutschland noch deutlich niedriger angesetzt werden. Jedoch ist der regulatorische Rahmen zwischen den OECD-Ländern sehr unterschiedlich, was die Vergleichbarkeit und Übertragbarkeit auf Deutschland einschränkt.

Der im Status quo in Deutschland vorzufindende Kapazitätsüberhang und die ökonomische Notwendigkeit der Fallzahlsteigerung in Kombination mit dem gestiegenen Qualitätsbewusstsein und Anspruchsverhalten der Patienten führen zu einem zunehmenden Konkurrenzdruck zwischen den Krankenhäusern (Hacker 2016; Bertelsmann Stiftung 2019; Dieterich et al. 2019). Neben der Zentralisierung und Verbundbildung versuchen die Krankenhäuser auch durch einen so genannten Innovationswettbewerb, d.h. die Vorhaltung von attraktiven Spezialzentren oder besonders minimalinvasiver Operationsverfahren, eine Steigerung der Patientenzahl zu generieren (Reifferscheid et al. 2012; Lüngen und Büscher 2012; Beivers 2010). So konkurrieren Krankenhäuser nicht nur auf der jeweiligen Versorgungsstufe miteinander, was Neubauer als horizontalen Wett- 


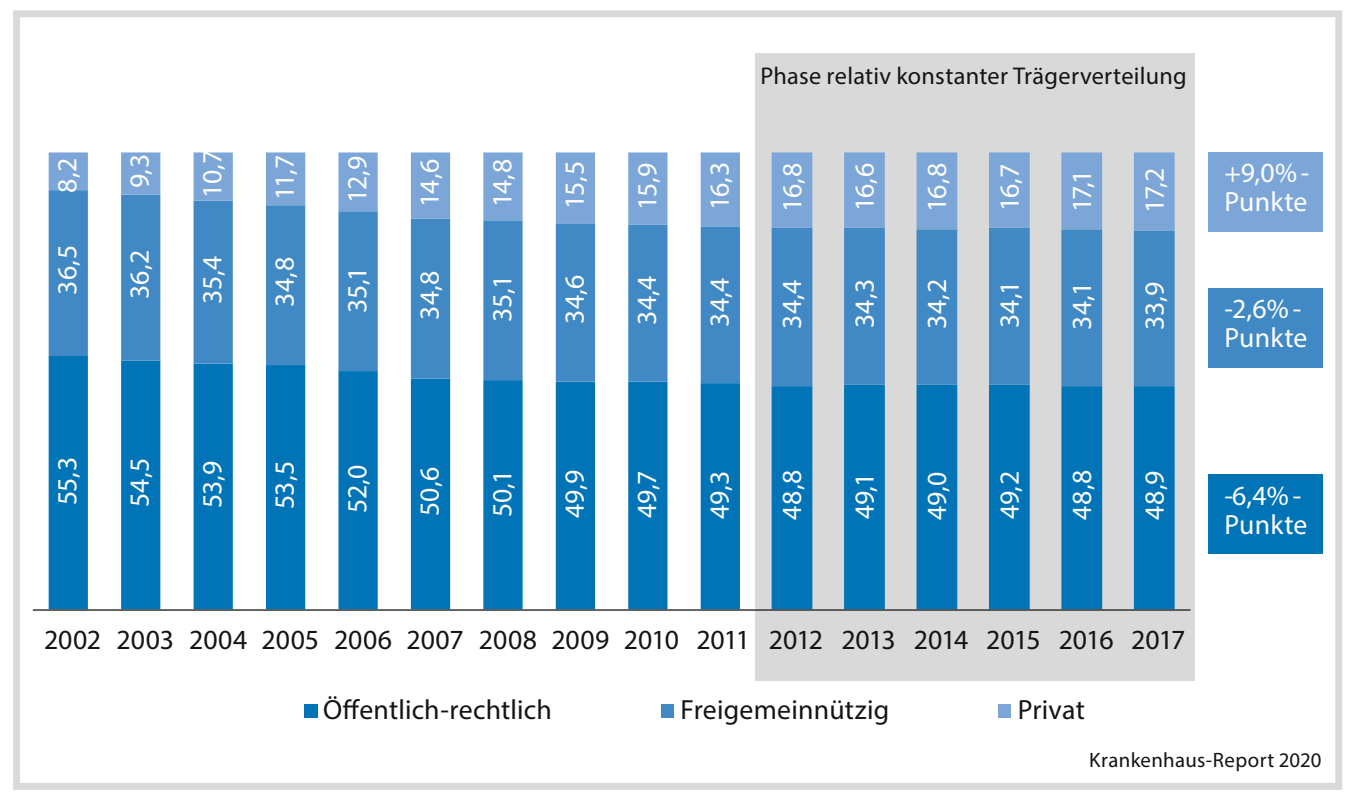

- Abb. 1.7 Marktanteil nach Trägerschaft gemessen in Zahl der Fälle (2002 bis 2017; in \%) (Quelle: RWI/hcb; Statistisches Bundesamt 2018) (Anmerkung: Alle Krankenhäuser)

bewerb bezeichnet, sondern auch auf unterschiedlicher Versorgungsstufe, dem so genannten vertikalen Wettbewerb (Neubauer 2002b; Neubauer und Beivers 2010; Neubauer et al. 2011). Somit unterwandern die Patienten die staatlich vorgesehene Krankenhaushierarchieplanung, was diese - zumindest zum Teil obsolet erscheinen lässt. Durch den vertikalen Wettbewerb kommt es darüber hinaus zu einer Vorhaltung von Doppelkapazitäten.

Zusammenfassend lässt sich feststellen, dass das Ziel einer höheren Wettbewerbsintensität zwischen den Krankenhäusern zwar erreicht wurde, aber gleichzeitig nur zu einem unterproportionalen Abbau redundanter Strukturen geführt hat.

\subsubsection{Das Ziel der Förderung des Strukturwandels}

Die DRG-Einführung war so angelegt, dass es zu Gewinnern und Verlieren kommt (Dieterich et al. 2019), was indirekt zu einem Struktur- wandel der Krankenauslandschaft führen sollte. Inwiefern dieser Strukturwandel tatsächlich eingetreten ist und ob er zu gewünschten, neuen und bedarfsgerechten Versorgungsstrukturen geführt hat, ist kritisch zu hinterfragen.

Dies wirft die Frage nach einer Neujustierung bzw. einem ordnungspolitischen Anpassungsbedarf der G-DRG-Vergütungssystematik mit neuen Anreizen auf. (Roeder et al. 2007; Geissler et al. 2012; Braun et al. 2008). So tragen die Krankenhäuser unter DRG-Bedingungen die Kostenverantwortung für die Leistungserstellung und dürfen daher die wirtschaftlichen Aspekte nicht aus den Augen verlieren. Viele der sich potenziell durch den deutschen Weg der DRG-Einführung ergebenden Fehlanreize wurden schon in den 1990er-Jahren diskutiert. Sicherlich war und ist es ein hehres und vielleicht zu hoch gestecktes Ziel, primär mit der Einführung eines neuen Vergütungssystems die von der Gesundheitspolitik erkannten Strukturprobleme lösen zu wollen. Dies kann ein Fallpauschalensystem allein nicht erbringen. Nicht zuletzt deswegen hat der Gesetzgeber u. a. durch das KHSG und der Einführung 
des Strukturfonds $s^{5}$ versucht, den angesprochen Wandel in die politisch gewünschte Richtung zu lenken.

Beispielsweise wurden die Mittel des Strukturfonds bis dato stark nachgefragt. Gemessen an den Anträgen, die bei den Bundesländern eingingen, waren sie mehr als zweifach „überzeichnet". Bis Ende Mai 2018 wurden rund 80 \% der zur Verfügung stehenden Mittel vom Bundesversicherungsamt bewilligt. Dabei entfielen $5 \%$ auf die "Schließung", $6 \%$ auf „Umwandlung " und $89 \%$ auf „Konzentration“. Insgesamt beinhalten die bewilligten Projekte einen Abbau von 5.290 Betten. Davon werden 2.460 Betten an anderer Stelle wiederaufgebaut, sodass sich ein Netto-Abbau von 2.830 Betten ergibt. Die Mittel des Strukturfonds reichen aber nicht aus, um in Deutschland eine optimale Struktur der Krankenhäuser zu erreichen. Will man etwa die bereits erwähnte günstigere Struktur Sachsens erreichen, wären dafür ca. 11 Mrd. $€$ nötig. Bundesweit müssten dann rund 280 Standorte geschlossen werden. Tatsächlich erreicht der Strukturfonds davon bereits etwa ein Zehntel, was näherungsweise der Relation der derzeit eingesetzten Mittel zu den dafür insgesamt benötigten Mitteln entspricht. Die beschlossene Fortführung des Strukturfonds ist daher sinnvoll. Doch es gibt Verbesserungspotenzial. Ordnungspolitisch richtig wäre es, dafür Steuermittel einzusetzen statt Mittel aus dem Gesundheitsfonds. Zudem lieBe sich durch wettbewerbliche Elemente eine effizientere Allokation der Fondsmittel erreichen: Krankenhausträger sollten selbst in einem Wettbewerbsverfahren Anträge auf Mittel des Strukturfonds stellen können und diejenigen mit den besten Kosten-Nutzen-Relationen den Zuschlag erhalten (Augurzky et al. 2019).

5 Strukturfonds ( $\$ 12 a$ Krankenhausfinanzierungsgesetz (KHG) und §§ 11-18 KrankenhausstrukturfondsVerordnung (KHSFV)): Einmalförderung zum Abbau von stationären Kapazitäten, z. B. durch Schließung oder Zentralisierung.

\subsection{Herausforderungen für die Zukunft: Ordnungspolitisch sinnvolle Anpassungen des DRG-Systems}

Trotz der dargestellten Fehlentwicklungen hat es etwas mehr als zehn Jahre nach formaler DRG-Einführung gedauert, bis der Gesetzgeber zunächst mit Hilfe des KHSG versucht hat, bestehende Fehlanreize abzubauen und das System in einigen Bereichen neu zu justieren. So boten - wie bereits beschrieben - anscheinend einzelne DRGs vor dem KHSG einen Anreiz zur Mengenausweitung, trotz Mehrerlösausgleich und Mehrleistungsabschlägen (Mostert et al. 2012; Lüngen und Büscher 2012; Reifferscheid et al. 2012). Auch die längst überfällige Diskussion der Qualitätsmessung und -steuerung via Qualitätsindikatoren in Ergänzung zur DRG-Vergütung war und ist berechtigt (Beivers 2019b).

Diesen Themen hat sich in der letzten Legislaturperiode das KHSG angenommen und es wurde versucht, neben der Einführung des Fixkostendegressionsabschlags (FDA) auch mit einer Absenkung von Bewertungsrelationen für „mengenanfällige DRGs“, unabhängig von der Kalkulation, Steuerungseffekte zu erzielen. Allerdings handelt es sich bei Letzterem um einen Eingriff in die DRG-Kalkulation des InEK.

Die derzeitigen Reformbemühungen zur Anpassung des Fallpauschalensystems stellen hingegen einen schwerwiegenden und bedenklichen Eingriff dar und lassen die berechtigte Frage aufwerfen, ob hier der Einstieg in den Ausstieg aus dem Fallpauschalensystem geplant ist. Als großer ordnungspolitischer Irrweg muss das Vorhaben des sogenannten „Pflexit" beurteilt werden, wonach Pflegepersonalkosten künftig unabhängig von Fallpauschalen zu vergüten sind. Dies führt zu einer neuartigen Vergütungskombination, die auf DRG-Pauschalen (die um die Pflegepersonalkosten bereinigt werden) einerseits und auf einer Pflegepersonalkostenvergütung andererseits basiert (Leber 2019; Beivers 2019b). Bezogen auf die Pflegepersonalkosten ist dies ein Rückfall in das 
Selbstkostendeckungsprinzip der 1990er Jahre. Durch die Selbstkostendeckung im Krankenhaus schafft man starke Fehlanreize dafür, möglichst viele Aufgaben der Pflege zuzuordnen und Pflegekräfte aus anderen Segmenten ohne Selbstkostendeckung, wie z.B. aus der Reha und Altenpflege, abzuziehen. Eine langfristige und erforderliche Stärkung der Pflege in Deutschland bleibt dadurch aus und der bereits heute bestehende Fachkräftemangel dürfte sich unnötig weiter zuspitzen. Um die steigende Zahl an pflegebedürftigen Menschen in Zukunft nicht unversorgt zu lassen, muss der Beruf stattdessen attraktiver werden. Dies kann zum Beispiel durch die Übertragung von Verantwortung und neue Karrieremöglichkeiten geschehen, wie es im Ausland bereits stattfindet (Lehmann et al. 2019). Die Ausgliederung der Pflegekosten löst dieses Problem hingegen nicht und scheint vielmehr ein Versuch zu sein, einzelne - in der Tat existierende Probleme durch ein immer komplexer werdendes Vergütungssystem lösen zu wollen. De facto wird das Vergütungssystem dadurch aber handlungsunfähig und dient keinesfalls mehr zur Steuerung und zur Setzung von strukturund wegweisenden Anreizen. Vielmehr fehlt der ordnungspolitische Kompass. Findet keine Anpassung statt, besteht die Gefahr, das zugegebenermaßen anpassungsbedürftige deutsche Fallpauschalensystem am Ende zu zerstören. Auch seitens der Ärzteschaft gibt es die Forderung nach der Ausgliederung ihrer Personalkosten aus dem DRG-System. Wenn diese Entwicklung stattfindet, sind ca. 40 bis $50 \%$ der Kosten eines Krankenhauses der Selbstkostendeckung zugeordnet. Eine Pauschale für die übrigen Kosten scheint dann wenig zielführend. Daher sollte besser über sinnvolle Anpassungen nachgedacht werden.

\subsubsection{Mangelnde Finanzierung der Investitionskosten}

Das Thema der Neuregelung einer auskömmlichen Investitionsfinanzierung durch die Bun- desländer hat leider, wie schon zu oft, abermals keinen Einzug in die aktuellen Reformgesetze gefunden, obgleich verschiedene Lösungsoptionen bekannt und publiziert sind. Will man jedoch das derzeitige Vergütungssystem und dessen Fehlanareise anpassen, ist dies ohne Lösung der Investitionskostenproblematik schlichtweg nicht möglich. Viele Krankenhäuser sind im Status quo dazu gezwungen, durch Fallpauschalenerlöse ihre nicht geförderten Investitionskosten zu finanzieren, die dort jedoch in der Kalkulation nicht abgebildet sind. Will man daher die sich ergebenden Schieflagen und Fehlanreize durch das Fallpauschalensystem korrigieren (wie beispielsweise eine Fehlallokation der Ressourcen zu Lasten der Pflege oder den Anreiz zum Fallzahlwachstum), muss zunächst das Thema der Investitionskostenfinanzierung gelöst werden.

Wie erwähnt mangelt es an Vorschlägen nicht (Malzahn und Wehner 2010). Im Jahr 2017 wurden u. a. im Gutachten „Stand und Weiterentwicklung der Investitionsförderung im Krankenhausbereich" im Auftrag des Bundesministeriums für Gesundheit verschiedene Möglichkeiten (wie zinslose Investitionskredite, Vorsteuerabzugsvoucher oder Digital Boost) erarbeitet und auf ihre Umsetzbarkeit dahingehend geprüft, ob auch der Bund durch eine gezielte Förderung zur Schließung oder Reduktion der Förderlücke beitragen kann (Augurzky et al. 2017).

Die anstehenden gewaltigen Herausforderungen im Gesundheitswesen werden nicht allein dadurch gemeistert werden können, dass die Ablauf- und Aufbauorganisation der einzelnen Leistungserbringer immer weiter optimiert werden. Vielmehr werden dazu auch völlig neue effizienzsteigernde Innovationen nötig sein, wie Digitalisierung der Medizin, Telemedizin, künstliche Intelligenz oder auch Robotik. Da sie Zeit bis zur Marktreife brauchen, gilt es, die Digitalisierung im Gesundheitswesen energisch voranzutreiben. Länder wie Dänemark können hierbei als Vorbild dienen. Die Digitalisierung hat in der dortigen Krankenhausreform einen besonderen Stellenwert. Auch in Deutschland braucht 
es eine breit angelegte Digitalisierungsstrategie.

Dafür ist jedoch ausreichend Investitionskapital vonnöten, das aufgrund der bestehenden Förderlücke nicht zur Verfügung steht. Der aktuelle Krankenhaus Rating Report 2019 schätzt die Förderlücke auf etwa 2,6 Mrd. € jährlich (Augurzky et al. 2019). Zum Teil schließen die Krankenhäuser diese jährliche Investitionslücke aus eigener Kraft und versuchen auch, Investitionen im Bereich der Digitalisierung selbst zu finanzieren. Jedoch gelingt es den Kliniken nicht, die Förderlücke gänzlich zu schließen, sodass es zu einem schleichenden Substanzabbau und zu einem zu geringen Ausbau der Digitalisierung kommt (Augurzky et al. 2017; Augurzky und Beivers 2019). Beispielsweise sieht der vom RWI vorgeschlagene „Digital Boost" vor, Investitionen in die Digitalisierung durch ein befristetes Investitionsprogramm von acht Jahren zu fördern. Ziel ist, eine zeitgemäße IT-Infrastruktur und die elektronische Vernetzung der Krankenhäuser zu ermöglichen, wie sie in anderen Ländern bereits existiert.

\subsubsection{Ambulant und stationär}

Um integrierte, sektorenübergreifende Versorgungsprozesse sicherzustellen, muss ein Vergütungssystem die richtigen Anreize setzen (Güssow 2007). Die heutigen Fallpauschalen weisen kaum Anreize für eine intersektorale Versorgung auf - vielmehr stellen sie eine sektorenspezifische Vergütung dar. Infolgedessen wird lediglich die Leistungserstellung innerhalb des jeweiligen Sektors optimiert. Wünscht man hingegen eine sektorenübergreifende Optimierung, benötigt man auch integrierte Vergütungsmodelle (Güssow 2007). Dem Koalitionsvertrag der 19. Legislaturperiode folgend soll sich dieser Problematik die Bund-LänderArbeitsgruppe annehmen, die bis zum Jahr 2020 unter anderem Vorschläge für die Einführung einer sektorenübergreifenden Vergütung machen soll. Bei der Diskussion um das
Zusammenwachsen der Sektoren - sprich der Suche nach sektorenübergreifenden, hybriden Vergütungsmodellen - kommt man aber so lange nicht voran, bis der Gesetzgeber nicht klare Definitionen vorgibt. Die Hybrid-DRGIdee der Techniker Krankenkasse weist in die richtige Richtung. In zentralen Punkten bleiben die Hybrid-DRGs derzeit jedoch noch wichtige Antworten schuldig (Beivers 2018; Beivers und Neubauer 2017). Wichtig wäre eine juristisch und medizinisch klare und pragmatische Abgrenzung der einzelnen Leistungen zwischen ambulant, teil- und vollstationär, um nicht „Äpfel“ und „Birnen“ miteinander zu vergleichen. Wenn dies nämlich geschieht, kommt es zu einer nicht zielführenden „Mischfinanzierung", die das System und die Versorgung nicht verbessern wird.

Ein internationaler Vergleich, wie ihn u. a. Tan et al. 2014 oder auch Geissler et al. 2012 durchführen, zeigt, dass bereits positive Beispiele vorhanden sind. So sind in Schweden von rund 980 DRG nur rund zwei Drittel für den stationären Krankenhaussektor vorgesehen, das andere Drittel kommt im ambulanten Bereich und bei Behandlungen in der Tagesklinik zur Anwendung. Der aktuelle Kabinettsentwurf zum MDK-Reformgesetz sieht eine Erweiterung des Katalogs für ambulante Operationen und stationsersetzende Eingriffe für Krankenhäuser vor - löst aber das strukturelle Problem fehlender intersektoraler Vergütungssysteme leider nicht. Solange für die Krankenhäuser kein relevanter Anreiz besteht, wo immer möglich ambulant bzw. teilstationär $\mathrm{zu}$ arbeiten und sich dies für sie auch lohnt, wird hier wenig passieren (Beivers 2019a).

Eine Lösungsoption kann die Einführung von Capitation-Modellen sein, die eine sektorenübergreifende Vergütung darstellen. Mittelfristig sollten solche Modelle regional erprobt werden können, um Erfahrungen damit zu sammeln. Für definierte Regionen - mit etwa 200.000 bis 400.000 Einwohnern - sollten morbiditätsorientierte Regionalbudgets festgelegt werden, die möglichst viele Gesundheitsleistungen (mindestens aber die ambulante und stationäre Akutversorgung), klar definier- 
te Qualitätsstandards (Indikations-, Strukturund Prozessqualität) und messbare Ergebnisparameter umfassen. Die Leistungserbringer der Region entscheiden eigenständig darüber, wie sie die Mittel des Regionalbudgets einsetzen und wie sie die Behandlungen durchführen - ambulant oder stationär - oder ob sie verstärkt auf Prävention setzen, um Behandlungen $\mathrm{zu}$ vermeiden. Dabei muss stets gewährleistet sein, dass die Bevölkerung die freie Arztund Krankenhauswahl hat, sodass sie jederzeit auch Leistungserbringer anderer Regionen aufsuchen kann, falls sie mit der lokalen Versorgung unzufrieden ist. Leistungen, die ein $\mathrm{Pa}-$ tient in anderen Regionen in Anspruch nimmt, mindern dann das Regionalbudget der Region, in der der Patient lebt. Umgekehrt wirkt es budgeterhöhend, wenn Patienten aus anderen Regionen zuwandern. Eine vertiefende Ausarbeitung dazu findet sich in Augurzky et al. (2018b).

Langfristig könnten solche Capitation-Modelle mindestens in ländlichen Regionen die heutigen Vergütungssysteme ablösen. In städtischen Regionen mit einer hohen Wettbewerbsdichte wird es dagegen zunächst schwierig sein, für einen ausreichend großen Stadtteil ein Regionalbudget zu definieren, weil unklar ist, mit welchem Leistungserbringer bzw. Konsortium aus Leistungserbringern ein Vertrag geschlossen werden kann. Wenn die Konzentration der Krankenhauskapazitäten jedoch weiter anhält und eine effektive Einbindung der Vertragsärzte gelingt, dürfte dies im Laufe der Zeit auch in Städten einfacher funktionieren. In Madrid existiert beispielsweise für die Stadtteile um das Hospital Universitario Rey Juan Carlos bereits ein solches Modell, das von der Bevölkerung gut angenommen wird (Augurzky et al. 2019). Auch der Beitrag von Benstetter et al. in $>$ Kap. 5 dieses Krankenhaus-Reports untersucht die sich durch unterschiedlichen Capitation-Modelle ergebenden (Qualitäts-)Aspekte sowie die Resonanz der jeweiligen Bevölkerung.

\subsubsection{Vorhaltung}

DRGs bilden in Deutschland die zentrale Grundlage für einen Großteil der stationären Leistungsvergütung. Die spezifisch deutsche, hohe Qualität der Kalkulation lässt es sogar $\mathrm{zu}$, aufgrund der Kostenhöhe zu über $80 \%$ auf die medizinische Fallschwere rückschließen zu können (Beivers 2019b). Dies ist bemerkenswert. $\mathrm{Zu}$ den Implikationen und Auswirkungen von Fallpauschalen gibt es viele Untersuchungen und Thesen. Die Entwicklung der Fallzahlen gehört dabei zu den sehr kontrovers diskutierten Themen (Reifferscheid et al. 2012; Mostert et al. 2012; Lüngen und Büscher 2012; Geissler et al. 2012; Augurzky et al. 2012). Wie bereits dargestellt ist hingegen jüngst ein Rückgang des Casemix-Volumens zu verzeichnen, was als "Ende des Wachstums“ tituliert wurde. Vor dem Hintergrund, dass Krankenhäuser fixkostenintensive Gesundheitsdienstleistungen anbieten, die sich $u$. a. aus einer hohen Vorhalteleistung ergeben, scheint es wichtig, im Rahmen der Zukunft des DRG-Systems darüber nachzudenken, welche Leistungen zukünftig pauschal im Sinne einer Vorhaltungsleistung und welche Leistungen „per case“ $\mathrm{zu}$ finanzieren sind und damit partiell gänzlich neue Anreize zu setzen.

Im Bereich der Notfallversorgung oder im Rahmen der Sicherstellungszuschläge nach $\$ 17$ KHG wurde und wird dies bereits diskutiert. Bei Letzteren soll ein krankenhausbezogener Pauschalzuschlag gewährt werden, der über alle Fälle hinweg abgerechnet wird. Die Finanzierung der Sicherstellungszuschläge verbleibt aber auch nach dem KHSG bei den Krankenversicherungen. Es ist jedoch fraglich, inwiefern die Sicherstellung, die ja primär - bedingt durch die Vorhalteleistung - ein Fixkostenproblem ist, nicht eigentlich auch von den Ländern zumindest teilweise zu finanzieren ist, da dies eine originäre Aufgabe der Daseinsvorsorge ist. Die Sicherstellung und Finanzierung der Notfallversorgung, die der Idee des Bundesministeriums für Gesundheit (BMG) folgend zukünftig intersektoral durch sogenannte INZs (Integrierte Notfallzentren) sichergestellt 
werden soll, wirft hier ähnliche Fragen auf. Um bei der Finanzierung der INZs neue Fehlanreize zu vermeiden, sollte das INZ von einem sektorenunabhängigen Budget finanziert werden. Krankenhäuser könnten beispielsweise für die Fälle, die sie vom INZ zugewiesen bekommen, Abschläge auf die jeweiligen DRGs in das INZ-Budget abführen, da die Erstarbeit (Triagierung, Diagnosestellung etc.) für diese Fälle ja entfällt. Wichtig ist sicherzustellen, dass das INZ für seine jeweilige Leistung aufwandsgerecht, aber unabhängig von der jeweiligen Nachbehandlung der Patienten vergütet wird. Denkbar wären auch sogenannte HybridDRGs für diesen Bereich oder pauschale, fallzahlunabhängige Vergütungsmodelle.

Dies belegt die Notwendigkeit der Klärung, was im Krankenhaus- und Gesundheitssektor eine Vorhalteleistung darstellt und nicht „per case“ zu finanzieren ist. Folgerichtig muss dann auch beantwortet werden, wer diese Vorhalteleistungen zahlt: Die Daseinsvorsorge und damit der Staat - oder die Solidargemeinschaft der Versicherten. Daher ist diese Debatte von der Investitionskostenthematik untrennbar (Beivers 2019b).

\subsubsection{Indikationsqualität vs. Mengensteuerung}

Mit den derzeitigen gesundheitspolitischen Instrumenten der Mengensteuerung wird zwar versucht, bestehende Fehlanreize zu mindern, jedoch führt beispielsweise der FDA abermals zu neuen Fehlanreizen, beispielsweise hinsichtlich des Qualitätswettbewerbs und der Optimierung von Krankenhausstrukturen. Daher sollten alternative Mengensteuerungsinstrumente in Erwägung gezogen werden, wie sie auch Bäuml in $>$ Kap. 9 dieses KrankenhausReports beschreibt. Beispielsweise sind nicht nur die Leistungserbringer, sondern auch die Patienten stärker in ein Lösungskonzept einzubeziehen. Dies kann theoretisch durch die finanzielle Beteiligung in Form von Selbstbehalten bei elektiven Leistungen geschehen. Hel- fen kann aber auch eine bessere bzw. effektivere Ausgestaltung der Patientensteuerung. Zentral ist dabei ein stärkerer Fokus auf die Indikationsqualität. Zwar misst der Gesetzgeber dem Thema Qualität u. a. im KHSG eine zentrale Bedeutung bei, bleibt aber beim Thema der Indikationsqualität hinter den Erwartungen zurück. So scheint es äußerst fragwürdig, warum Leistungen, die bereits erbracht wurden, auf ihre Sinnhaftigkeit bzw. Notwendigkeit bspw. durch den Medizinischen Dienst der Krankenkassen ex post überprüft werden, anstatt zu Beginn der „Wertschöpfungskette“ zu hinterfragen, ob der Eingriff eigentlich medizinisch nötig bzw. sinnvoll ist (Beivers 2019b). Daher sollte sich die generelle Qualitätsdebatte im Krankenhauswesen wesentlich stärker auf das Thema der Indikationsqualität fokussieren, um somit auch Lösungen für eine sinnvolle Mengensteuerung zu finden. Vorschläge durch das IQTIG (Institut für Qualitätssicherung und Transparenz im Gesundheitswesen) wären hier sehr wünschenswert.

\subsection{Ausblick}

Die DRG-Einführung ist in vielerlei Hinsicht eine Erfolgsgeschichte, jedoch offenbaren sich auch immer mehr Probleme. Manche Ziele wurden erreicht, andere verfehlt und neue Fehlanreize geschaffen. Daher ist es an der Zeit, das System - und zwar stärker als bisher geschehen - so anzupassen, dass es seine gewünschten Wirkungen entfalten kann. Zentrale Punkte sind dabei die Lösung der Investitionskostenproblematik, die Etablierung sektorenübergreifender, hybrider Vergütungsmodelle wie auch die Klarstellung, welche Bereiche der Vorhaltung pauschal und welche Bereiche der Versorgung "per case“ zu finanzieren sind. Hinzu kommt die generelle Frage, ob man bei der Diskussion um "Qualität und Menge“ im DRG-System der Indikationsqualität nicht einen größeren Stellenwert einräumen sollte. Capitation-Modelle können eine weitreichende und interessante neue Vergütungsoption für gewisse Regionen darstellen. 
Im Zuge dieser Diskussion darf jedoch nicht vergessen werden, dass die jetzigen DRGFallpauschalen ein Vergütungssystem darstellen. Eine Überfrachtung der Anforderungen an dieses System, etwa politische Vorstellungen wie den Strukturwandel durchzusetzen, kann damit alleine nicht gelingen. Hinzu kommt, dass mit der DRG-Einführung bewusst auf mögliche Steuerungswirkungen eines Preissystems verzichtet wurde. Auch wenn der Beitrag des Öfteren die Begriffe „Preise“ und „Vergütungen" verwendet, unterscheiden sich beide Begriffe durchaus deutlich voneinander. Im deutschen DRG-System existieren genau betrachtet keine Preise, sondern nur Vergütungen. Krankenhäuser verzehren bei der Leistungserstellung Ressourcen, die ihnen wieder zugeführt werden müssen, sofern eine dauerhafte Leistungserstellung gewünscht wird (Neubauer und Beivers 2010). Diese Ressourcenzuführung wird allgemein auch als Vergütung oder Entgeltung bezeichnet und ist nicht Teil eines wettbewerblichen Marktes, auf dem Preise herrschen. Jedoch war und ist es eins der zentralen Ziele der DRG-Einführung in Deutschland, den Wettbewerb der Krankenhäuser untereinander zu fördern. Wettbewerb ohne Preise ist jedoch - der mikroökonomischen Theorie folgend - kaum möglich. Die DRG-Fallpauschalen könnten, in Kombination mit krankenhausindividuellen Basisfallwerten, eine gute Basis für einen geregelten, preislichen Wettbewerb bilden, ohne dabei in das System der Selbstkostendeckung zurückzufallen. In Deutschland dienen DRGs derzeit jedoch vorwiegend als Abrechnungseinheiten. Das Schlagwort lautet „gleiche Leistung, gleicher Preis“, wie im Rahmen der Bundesbasisfallwert-Diskussion formuliert. Dabei wird unter gleicher Leistung die gleiche DRG-Eingruppierung verstanden und daraus der Anspruch auf eine bundesweit einheitliche Vergütung abgeleitet. Die DRG-Eingruppierung ist tatsächlich jedoch zunächst allenfalls eine Gleichstellung der Patienten aus medizinisch-technischer Sicht.

Weitgehend unberücksichtigt bleiben, ob das Behandlungsziel erreicht wurde, sowie die zeitliche und örtliche Verfügbarkeit der Leistung. Aber auch Unterschiede in der lokalen Versorgungslage schlagen sich üblicherweise in Preisen nieder. Alle letztgenannten Faktoren werden im DRG-System ausgeblendet. Dies führt daher zu einer Unter-, Über- und Fehlversorgung im Krankenhausbereich, die sich mehr und mehr bemerkbar macht. Will man jedoch mit DRGs die Versorgung steuern, kommt man um eine gewisse geregelte Preissteuerung nicht herum. Es wäre daher sinnvoll, das DRG-Vergütungssystem möglichst nah einem Preissystem anzunähern, um die Vorteile eines Preissystems zu nutzen. Aus gesundheitsökonomischer wie auch aus ordnungspolitischer Sicht erscheint dieser Weg sinnvoller als vereinzelte dirigistische Eingriffe (Beivers 2019b), die zum Teil auch zu nicht nachhaltigen Investitionen und damit zur Verschwendung der ohnehin knappen investiven Mittel führen (z. B. Aufbau redundanter bzw. nicht sinnvoller Strukturen, um weiterhin Notfälle behandeln zu können). Vielleicht wäre es sinnvoller, die Kraft und Mühe, die man gerade für den „Pflexit“ aufbringt, in eine zukunftsweisende Neujustierung des DRG-Systems zu investieren. Das könnte eine große Anzahl an Problemen lösen.

Danksagung Wir danken Herrn Prof. Dr. Augurzky und Frau Dr. Michaela Lemm für ihre wertvollen Anmerkungen.

\section{Literatur}

Augurzky B, Beivers A (2019) Digitalisierung und Investitionsfinanzierung. In: Klauber J, Geraedts M, Friedrich J, Wasem J (Hrsg) Krankenhaus-Report 2019, Schwerpunkt: Das digitale Krankenhaus. Springer, Berlin, S 67-80

Augurzky B, Felder S, Wasem J (2012) Mengensteuerung über das G-DRG-Preissystem. In: Klauber J, Geraedts M, Friedrich J, Wasem J (Hrsg) Krankenhaus-Report 2013, Schwerpunkt: Mengendynamik: mehr Menge, mehr Nutzen? Schattauer, Stuttgart, S 175-187

Augurzky B, Bünnings C, Dördelmann S, Greiner W, Hein L, Scholz S, Wübker A (2016) Die Zukunft der Pflege im Krankenhaus. RWI Leibniz-Institut für Wirtschaftsforschung, Essen. http://www.rwi-essen.de/media/ 
content/pages/publikationen/rwi-materialien/rwimaterialien_104.pdf. Zugegriffen: 4. Okt. 2019

Augurzky B, Beivers A, Emde A, Halbe B, Pilny A, Straub N (2017) Stand und Weiterentwicklung der Investitionsförderung im Krankenhausbereich. Gutachten im Auftrag des Bundesministeriums für Gesundheit. RWI Leibniz-Institut für Wirtschaftsforschung, Essen. https://www.bundesgesundheitsministerium.de/ fileadmin/Dateien/5_Publikationen/Ministerium/ Berichte/Gutachten_Investitionsfoerderung_ Krankenhausbereich.pdf. Zugegriffen: 4. Okt. 2019

Augurzky B, Beivers A, Pilny A (2018a) Krankenhäuser in privater Trägerschaft 2018. Gutachten im Auftrag des Bundesverbandes Deutscher Privatkliniken, RWI Leibniz-Institut für Wirtschaftsforschung, Essen. http://www.rwi-essen.de/media/content/pages/ publikationen/rwi-materialien/rwi-materialien_ 122.pdf. Zugegriffen: 4. Okt. 2019

Augurzky B, Graf C, Griewing B, Walter D (2018b) Versorgung und Vergütung regional gedacht: "Von der Volumen- zur Wertorientierung". GuS 72(4-5):64-71

Augurzky B, Krolop S, Mensen A, Pilny A, Schmidt CM, Wuckel C (2019) Krankenhaus Rating Report 2019 - Das Ende des Wachstums? medhochzwei, Heidelberg

Baum G (2009) Zwischenbilanz aus Sicht der DKG. In: Roeder N, Hensen P, Rau F (Hrsg) Auswirkungen der DRG-Einführung in Deutschland - Standortbestimmung und Perspektiven. Kohlhammer, Stuttgart, S 25-28

Beivers A (2010) Ländliche Krankenhausversorgung in Deutschland: Eine gesundheitsökonomische Analyse. Europäische Hochschulschriften. Peter Lang, Frankfurt am Main

Beivers A (2018) Hybrid-DRGs: Äpfel und Birnen. Gesundheit und Pflege. Rechtszeitschrift Für Das Gesamte Gesundheitswes 5:161-164

Beivers A (2019a) Kommentar zum Orientierungswert: Auf ein Neues: Die Prüfung der Prüfungsreform. Bibliomed, Melsungen. https:// www.bibliomedmanager.de/news-des-tages/ detailansicht/38609-auf-ein-neues-die-pruefungder-pruefungsreform/. Zugegriffen: 4. Okt. 2019

Beivers A (2019b) Entgeltsystem-Reform: Mehr Ordnung bitte!". F\&w Führen Wirtschaften Im Krankenh 4:308311

Beivers A, Neubauer G (2017) Hybrid-DRGS: Die Richtung stimmt. Führen Wirtschaften 2:154-158

Beivers A, Waehlert L (2018) Steuerung der Mengendynamik nach dem KHSG: Implikationen für die Krankenhäuser. In: Da-Cruz P, Rasche C, Pfannstiel M (Hrsg) Entrepreneurship im Gesundheitswesen - Geschäftsmodelle, Prozesse, Funktionen, Bd. 2. Springer, Berlin

Bertelsmann Stiftung (Hrsg) (2017) Pflegepersonal im Krankenhaus: Mehr Pflegepersonal erhöht die Versorgungsqualität - Konkrete Vorgaben zum
Stellenplan sind erforderlich. Faktencheck Gesundheit, Daten, Analysen, Perspektiven, Nr. 6. Gütersloh. https://faktencheck-gesundheit.de/fileadmin/files/ BSt/Publikationen/GrauePublikationen/VV_FC_ Pflegepersonal_final.pdf. Zugegriffen: 4. Okt. 2019

Bertelsmann Stiftung (Hrsg) (2019) Zukunftsfähige Krankenhausversorgung: Simulation und Analyse einer Neustrukturierung der Krankenhausversorgung am Beispiel einer Versorgungsregion in NordrheinWestfalen. Gütersloh. https://www.bertelsmannstiftung.de/fileadmin/files/BSt/Publikationen/ GrauePublikationen/VV_Bericht_KH-Landschaft_ final.pdf. Zugegriffen: 4. Okt. 2019

Braun T, Rau F, Tuschen KT (2008) Die DRG-Einführung aus gesundheitspolitischer Sicht: Eine Zwischenbilanz. In: Klauber J, Robra B-P, Schellschmidt H (Hrsg) Schwerpunkt: Krankenhausvergütung - Ende der Konvergenzphase. Krankenhaus-Report 2007. Schattauer, Stuttgart, S 3-22

Bundesministerium für Gesundheit (BMG) (1990) Erprobung der Fallklassifikation: Patient Management Categories. Schriftenreihe des BMG, Bd. 81. Nomos, Baden-Baden

Bundesministerium für Gesundheit (BMG) (2019) KJ 1-Statistik: Gesetzliche Krankenversicherung: endgültige Rechnungsergebnisse, Gesundheitsberichterstattung des Bundes. BMG, Bonn

Bündnis Krankenhaus statt Fabrik (2018) Fakten und Argumente zum DRG-System und gegen die Kommerzialisierung der Krankenhäuser, 3. Auflage. März 2018, Maintal. https://www.krankenhaus-stattfabrik.de/196. Zugegriffen: 4. Okt. 2019

Deutscher Ethikrat (2016) Patientenwohl als ethischer Maßstab für das Krankenhaus. Stellungnahme, 5. April 21016, Berlin. https://www.ethikrat.org/ fileadmin/Publikationen/Stellungnahmen/deutsch/ stellungnahme-patientenwohl-als-ethischermassstab-fuer-das-krankenhaus.pdf. Zugegriffen: 4. Okt. 2019

Dieterich A, Braun B, Gerlinger T, Simon M (Hrsg) (2019) Geld im Krankenhaus: Eine kritische Bestandsaufnahme des DRG-Systems. Springer VS, Wiesbaden

Ernst \& Whinney (1986) Vorstudie zu diagnosebezogenen Fallpauschalen: Dokumentation, Analyse und Bewertung ausländischer Vorerfahrungen. Gesundheitsforschung, Bd. 143. Bundesministerium für Arbeit und Sozialordnung, Bonn

Friedrich J, Leber W-D, Wolff J (2010) Basisfallwerte zur Preis- und Produktivitätsentwicklung stationärer Leistungen. In: Klauber J, Geraedts M, Friedrich J (Hrsg) Krankenhaus-Report 2010, Schwerpunkt: Krankenhausversorgung in der Krise? Schattauer, Stuttgart, S 127-147

Geissler A, Wörz M, Busse R (2010) Deutsche Krankenhauskapazitäten im internationalen Vergleich. In: Klauber J, Geraedts M, Friedrich J (Hrsg) Krankenhaus-Report 2010, Schwerpunkt: Kran- 
kenhausversorgung in der Krise? Hrsg), Bd. 2010. Schattauer, Stuttgart, S 25-40

Geissler A, Scheller-Kreinsen D, Quentin W, Busse R (2012) DRG-Systeme in Europa - Anreize, Ziele und Unterschiede in zwölf Länder. Bundesgesundheitsblatt 55(5):633-642

Güssow J (2007) Vergütung Integrierter Versorgungsstrukturen im Gesundheitswesen: Weiterentwicklung pauschaler Vergütungsansätze zur Förderung prozessorientierter Strukturen unter besonderer Berücksichtigung der Krankenhausperspektive. Springer, Berlin

Hacker J (Hrsg) (2016) Zum Verhältnis von Medizin und Ökonomie im deutschen Gesundheitssystem: 8 Thesen zur Weiterentwicklung zum Wohle der Patienten und der Gesellschaft. Leopoldina, Nationale Akademie der Wissenschaften, Oktober 2016, Diskussion Nr. 7, Halle. https://www.leopoldina.org/uploads/ tx_leopublication/Leo_Diskussion_Medizin_und_ Oekonomie_2016.pdf

Kimberly JR, de Pouvourville G (Hrsg) (1993) The Migration of Managerial Innovation: Diagnosis-Related Groups und Health Care Administration in Western Europe. Jossey-Bass, San Francisco

Leber W-D (2019) Integrale Vergütung. F\&w Führen Wirtschaften Im Krankenh 3:208-210

Leber W-D, Scheller-Kreinsen D (2014) Teure Erfolgsgeschichte - 10 Jahre G-DRG-System. F\&w Führen Wirtschaften Im Krankenh 1:28-31

Lehmann Y, Schaepe C, Wulff I, Ewers M (2019) Pflege in anderen Ländern - Vom Ausland lernen? Stiftung Münch, medhochzwei, Heidelberg

Lüngen M, Büscher $G$ (2012) Mengensteigerungen in der stationären Versorgung: Wo liegt die Urache? In: Klauber J, Geraedts M, Friedrich J, Wasem J (Hrsg) Krankenhaus-Report 2013, Schwerpunkt: Mengendynamik: mehr Menge, mehr Nutzen? Schattauer, Stuttgart, S 83-93

Malzahn J, Wehner C (2010) Zur Lage der Investitionsfinanzierung der Krankenhäuser - Bestandsaufnahme und Reformbedarf. In: Klauber J, Geraedts M, Friedrich J (Hrsg) Krankenhaus-Report 2010, Schwerpunkt: Krankenversorgung in der Krise? Schattauer, Stuttgart, S 107-125

Mostert C, Leclerque G, Friedrich J (2012) Eckdaten der Leistungsentwicklung im Krankenhausmarkt 2011. In: Klauber J, Geraedts M, Friedrich J, Wasem J (Hrsg) Krankenhaus-Report 2013, Schwerpunkt: Mengendynamik: mehr Menge, mehr Nutzen? Schattauer, Stuttgart, S 21-43

Müller M-L (2009) Zwischenbilanz aus Sicht des Deutschen Pflegerates. In: Roeder N, Hensen P, Rau $F$ (Hrsg) Auswirkungen der DRG-Einführung in Deutschland - Standortbestimmung und Perspektiven. Kohlhammer, Stuttgart, S 32-36

Neubauer G (1993) Auf dem Weg vom Pflegesatz zur Fallpauschale. Führen Wirtsch Krankenh 10(1):38-40
Neubauer G (2002a) Auswirkungen eines DRG-basierten Vergütungssystems auf den Wettbewerb der Krankenhäuser. In: Wille E (Hrsg) Anreizkompatible Vergütungssysteme im Gesundheitswesen, Bd. 38. Nomos, Baden-Baden, S 159-176

Neubauer G (2002b) Auswirkungen der DRGs aus der Perspektive der Gesundheitsökonomie. In: Balzer K, Walter M (Hrsg) Zukunftsperspektiven in der Gefäßchirurgie. Steinkopff, Darmstadt, S 105-117

Neubauer G (2003a) Zur ökonomischen Steuerung der Krankenhausversorgung unter DRG-Fallpauschalen. In: Klauber J, Robra B-P, Schellschmidt H (Hrsg) Krankenhaus-Report 2003, Schwerpunkt: G-DRGs im Jahre 1. Schattauer, Stuttgart, S 101-119

Neubauer G (2003b) Ordnungspolitische Neuorientierung der Krankenhausversorgung auf der Basis von diagnosebezogenen Fallpauschalen. In: Klusen N, Straub C (Hrsg) Baden-Baden. Bausteine für ein neues Gesundheitswesen, Bd. 6. Nomos, In, S 91-107

Neubauer G, Beivers A (2010) Die Leistungen müssen die Vergütung bestimmen: Ein Plädoyer für das ordnungspolitische Resetting des DRG-Systems. F\&w Führen Wirtschaften Im Krankenh 01:38-42

Neubauer G, Unterhuber H (1987) Ökonomische Beurteilung der Preisfindung im DRG-Konzept. Krankenhaus 79(4):155-159

Neubauer G, Demmler G, Rehrmann P (1992) Erprobung der Fallklassifikation "patient management categories"für Krankenhauspatienten; Ergebnisbericht. Gutachten im Auftrag des Bundesministeriums für Gesundheit. Nomos, Baden-Baden

Neubauer G, Beivers A, Paffrath D (2011) Die Zukunft der Vergütung von Krankenhausleistungen. In: Klauber J, Geraedts M, Friedrich J, Wasem J (Hrsg) Schwerpunkt: Qualität durch Wettbewerb. KrankenhausReport 2011. Schattauer, Stuttgart, S 149-160

Reifferscheid A, Thomas D, Wasem J (2012) Zehn Jahre DRG-System in Deutschland - Theoretische Anreizwirkungen und empirische Evidenz. In: Klauber J, Geraedts M, Friedrich J, Wasem J (Hrsg) Krankenhaus-Report 2013, Schwerpunkt: Mengendynamik: mehr Menge, mehr Nutzen? Schattauer, Stuttgart, S 3-17

Rheinisch-Westfälisches Institut für Wirtschaftsforschung (RWI) (2012) Mengenentwicklung und Mengensteuerung stationärer Leistungen. Endbericht. RWI Projektbericht Forschungsprojekt im Auftrag des GKV-Spitzenverbandes (Hrsg), Essen

Roeder N, Bunzemeier H, Fiori W (2007) Ein lernendes Vergütungssystem Vom Budgetierungsinstrument zum deutschen Preissystem. In: Klauber J, Robra B-P, Schellschmidt H (Hrsg) Krankenhaus-Report 2007, Schwerpunkt: Krankenhausvergütung - Ende der Konvergenzphase. Schattauer, Stuttgart, S 23-47

Roeder N, Hensen P, Rau F (Hrsg) (2009) Auswirkungen der DRG-Einführung in Deutschland - Standortbestimmung und Perspektiven. Kohlhammer, Stuttgart 
Sachverständigenrat für die Konzertierte Aktion im Gesundheitswesen (SVR) (1992) Ausbau in Deutschland und Aufbruch nach Europa. Jahresgutachten. Nomos, Baden-Baden

Schreyögg J, Bäuml T, Krämer J, Dette T, Busse R, Geissler A (2014) Endbericht, Forschungsauftrag zur Mengenentwicklung nach $\S 17 \mathrm{~b}$ Abs. 9 KHG, Juli 2014, Hamburg Center for Health Economics, Hamburg. https://www. gkv-spitzenverband.de/media/dokumente/ krankenversicherung_1/krankenhaeuser/ budgetverhandlungen/mengensteuerung/ Gutachten_zur_Mengenentwicklung.pdf. Zugegriffen: 12. Jan. 2017

v. Stackelberg J-M (2009) Zwischenbilanz aus Sicht gesetzlichen Krankenversicherung. In: Roeder $\mathrm{N}$, Hensen P, Rau F (Hrsg) Auswirkungen der DRGEinführung in Deutschland, Standortbestimmung und Perspektiven. Kohlhammer, Stuttgart, S 29-31
Statistisches Bundesamt (Hrsg) (2018) Grunddaten der Krankenhäuser 2017. Fachserie 12: Gesundheitswesen, Reihe 6.1.1. Statistisches Bundesamt, Wiesbaden

Tan S, Geissler A, Serdén L, Heurgren M, van Inevel M, Redekop W, Hakkaart-van Roijen L (2014) DRG systems in Europe: variations in cost accounting systems among 12 countries. Eur J Public Health 24(6):10231028

Tuschen K-H (2007) Das DRG-System 2007. In: Roeder N, Bunzemeier H (Hrsg) Kompendium zum DRG System 2007, Band IV. Deutsche Krankenhausverlagsgesellschaft, Düsseldorf

Waehlert L, Beivers A, Auhuber TC (2015) Ordnungspolitische Herausforderungen und Handlungsbedarfe für die Versorgungsstruktur und Vergütung von Krankenhäusern: Ansatzpunkte zur Verknüpfung von Qualität und Wirtschaftlichkeit. In: Mülheims L et al (Hrsg) Handbuch Sozialversicherungswissenschaft. Springer, Wiesbaden, S 455-469

Open Access Dieses Kapitel wird unter der Creative Commons Namensnennung 4.0 International Lizenz (http:// creativecommons.org/licenses/by/4.0/deed.de) veröffentlicht, welche die Nutzung, Vervielfältigung, Bearbeitung, Verbreitung und Wiedergabe in jeglichem Medium und Format erlaubt, sofern Sie den/die ursprünglichen Autor(en) und die Quelle ordnungsgemäß nennen, einen Link zur Creative Commons Lizenz beifügen und angeben, ob Änderungen vorgenommen wurden.

Die in diesem Kapitel enthaltenen Bilder und sonstiges Drittmaterial unterliegen ebenfalls der genannten Creative Commons Lizenz, sofern sich aus der Abbildungslegende nichts anderes ergibt. Sofern das betreffende Material nicht unter der genannten Creative Commons Lizenz steht und die betreffende Handlung nicht nach gesetzlichen Vorschriften erlaubt ist, ist für die oben aufgeführten Weiterverwendungen des Materials die Einwilligung des jeweiligen Rechteinhabers einzuholen. 